\title{
Electromagnetic boundary conditions in differential-form formalism
}

\author{
I V Lindell $\dagger$ and B Jancewicz $\ddagger$ \\ $\dagger$ Electromagnetics Laboratory, Helsinki University of Technology, PO Box 3000, FIN-02015 \\ HUT, Espoo, Finland \\ \$Institute of Theoretical Physics, University of Wroclaw, pl. Maksa Borna 9, PL-50-204, \\ Wroclaw, Poland
}

Received 5 July 1999

\begin{abstract}
A simple way of deriving the electromagnetic boundary conditions in the differentialform formalism is outlined. The derivation is based on glueing together two independent electromagnetic source-field systems, each existing in an arbitrary electromagnetic environment. The combined electromagnetic source-field system contains a surface separating regions of the two original systems and media. It is shown that, in general, additional sources at the surface are needed for the new system to satisfy the Maxwell equations. This requirement is seen to create a set of boundary conditions at the surface. As a by-product, Huygens' principle can be simply formulated in differential form formalism.
\end{abstract}

\section{Introduction}

The set of equations known today as the Maxwell equations was not originally given by Maxwell in his Treatise [1]. In fact, his set of electromagnetic equations consisted of twenty scalar equations with twenty unknowns involving scalar components of both electromagnetic fields and potentials. The complexity in expression may have been the reason why there was only a handful of Maxwell's contemporaries who could really understand his theory, which again might have been the reason why it took a quarter of century to find experimental verification of the theory. Oliver Heaviside was first to express the Maxwell equations in a more compact vector form, basically that most often taught to physics and engineering students. Heaviside discarded the potentials and wrote two symmetric curl equations for the electric and magnetic field vectors, which he called the duplex method [2]. Meanwhile, vector analysis was developed to the present form by Gibbs [3].

To obtain a still more compact representation, the differential-form notation can be used. While the formalism, based on the exterior product by Grassmann, had already been introduced early this century by Elie Cartan, its application to electromagnetic theory has met with difficulties. Only since the 1970s have there been several attempts to suggest its use in electromagnetic theory. Inspired by books on applied mathematics [4-6], Deschamps was the first to write a thorough paper [7] which demonstrated the compactness of using differential forms in electromagnetic theory, when compared with the Gibbsian vector notation. A few other papers [8-13] and a book [14] followed on the same subject. So far, the formalism is still overshadowed by vector calculus in spite of the simple geometrical content of the differential-form quantities.

One of the reasons why differential-form notation has not been adopted in classroom use is that all aspects of electromagnetism have not yet been efficiently treated in terms of differential 
forms. In the present study it is shown how the electromagnetic boundary conditions can be derived very simply through the differential-form formalism. The method applied here is somewhat similar to that discussed in [11] and also invokes [15]. However, while the starting point in [11] is a discontinuity in the electromagnetic field, the present approach starts from combining two distinct field problems. This has the additional advantage that Huygens' principle can be introduced as a by-product.

\section{Maxwell equations}

The present text requires some background knowledge on multivector algebra and differentialform formalism, e.g., according to [4,6,7]. We follow the notation of [7] except that the outer product is explicitly expressed in terms of the wedge sign $\wedge$ and the magnetic sources are not neglected (magnetic sources are here considered as equivalent sources without expressing any opinion on the existence of magnetic poles). In four-dimensional differential-form notation, the Maxwell equations can be expressed in compact form as

$$
\mathbf{d} \wedge \Psi=\gamma \quad \mathbf{d} \wedge \boldsymbol{\Phi}=\tau .
$$

Here, $\boldsymbol{\Psi}$ and $\boldsymbol{\Phi}$ are the electromagnetic field two-forms and they can be expanded as

$$
\mathbf{\Psi}=\mathbf{D}-\mathbf{H} \wedge \mathbf{d} t \quad \mathbf{\Phi}=\mathbf{B}+\mathbf{E} \wedge \mathbf{d} t
$$

where $\mathbf{D}$ and $\mathbf{B}$ are the electric and magnetic two-forms, and $\mathbf{E}$ and $\mathbf{H}$ are the electric and magnetic one-forms. The electric and magnetic source three-forms $\gamma, \tau$ can be expanded as

$$
\gamma=\varrho-\mathbf{J} \wedge \mathbf{d} t \quad \tau=\varrho_{\mathrm{m}}-\mathbf{M} \wedge \mathbf{d} t
$$

where $\varrho$ and $\varrho_{\mathrm{m}}$ are the electric and magnetic charge density three-forms, and $\mathbf{J}$ and $\mathbf{M}$ are the electric and magnetic current density two-forms.

The operator $\mathbf{d}$ of the differential-form formalism corresponds to the $\nabla$ operator of vector analysis and $\mathbf{d} t$ corresponds to the unit vector along the time axis. The wedge multiplication sign $\wedge$ corresponds to the cross or dot multiplication of vector analysis, depending on the grade of the two factors. It is quite easy to transfer the present derivation to the language of Gibbsian vector analysis.

\section{Combining source-field systems}

Let us consider two independent electromagnetic systems of sources and their fields $\left\{\boldsymbol{\Phi}_{i}, \boldsymbol{\Psi}_{i}, \gamma_{i}, \boldsymbol{\tau}_{i}\right\}$ with $i=1,2$, which exist in respective electromagnetic media 1 and 2 each filling the whole space. Assuming a non-moving surface $S$, open or closed, separating the space into two regions $V_{1}$ and $V_{2}$ (see figure 1), let us create a new electromagnetic system $\{\boldsymbol{\Phi}, \Psi, \gamma, \tau\}$ by taking the part of system 1 in $V_{1}$ and the part of system 2 in $V_{2}$ and glueing them along the surface $S$. This can be done mathematically through two characteristic functions $P_{1}(\mathbf{r})$ and $P_{2}(\mathbf{r})$ satisfying $P_{1}(\mathbf{r})+P_{2}(\mathbf{r})=1$ and

$$
\begin{array}{llll}
P_{1}(\mathbf{r})=1 & \mathbf{r} \in V_{1} & P_{1}(\mathbf{r})=0 & \mathbf{r} \in V_{2} \\
P_{2}(\mathbf{r})=0 & \mathbf{r} \in V_{1} & P_{2}(\mathbf{r})=1 & \mathbf{r} \in V_{2} .
\end{array}
$$

On the surface $S$ we can assume $P_{1}(\mathbf{r})=P_{2}(\mathbf{r})=1 / 2$. The media in spaces 1 and 2 do not have any restrictions, they may be isotropic, anisotropic, linear or nonlinear. The combination source-field system is now defined by

$$
\begin{aligned}
& \boldsymbol{\Phi}(\mathbf{r})=\Phi_{1}(\mathbf{r}) P_{1}(\mathbf{r})+\Phi_{2}(\mathbf{r}) P_{2}(\mathbf{r}) \\
& \Psi(\mathbf{r})=\Psi_{1}(\mathbf{r}) P_{1}(\mathbf{r})+\Psi_{2}(\mathbf{r}) P_{2}(\mathbf{r}) \\
& \gamma(\mathbf{r})=\gamma_{1}(\mathbf{r}) P_{1}(\mathbf{r})+\gamma_{2}(\mathbf{r}) P_{2}(\mathbf{r})+\gamma_{\mathrm{s}}(\mathbf{r}) . \\
& \tau(\mathbf{r})=\tau_{1}(\mathbf{r}) P_{1}(\mathbf{r})+\tau_{2}(\mathbf{r}) P_{2}(\mathbf{r})+\tau_{\mathrm{s}}(\mathbf{r}) .
\end{aligned}
$$




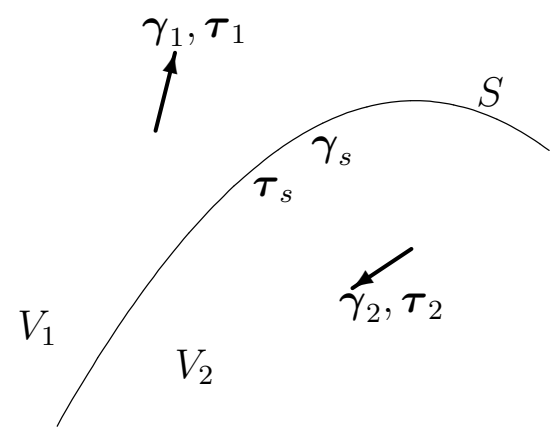

Figure 1. Surface $S$ separates two electromagnetic source-field systems 1 and 2. Satisfaction of the Maxwell equations requires additional surface sources at $S$.

Here we have added extra source terms $\gamma_{\mathrm{s}}(\mathbf{r}), \tau_{\mathrm{s}}(\mathbf{r})$ in case they are needed to make the combination system satisfy the Maxwell equations. Also, we have tacitly assumed that the original sources 1 and 2 have no delta singularities on $S$.

Let us now require that the system $\{\boldsymbol{\Phi}, \Psi, \gamma, \tau\}$ satisfies the Maxwell equations (1). Inserting equations (6)-(9), we have

$$
\begin{aligned}
\mathbf{d} \wedge \boldsymbol{\Phi}(\mathbf{r})-\tau & =\left[\mathbf{d} \wedge \boldsymbol{\Phi}_{1}(\mathbf{r})\right] P_{1}(\mathbf{r})+\left[\mathbf{d} \wedge \Phi_{2}(\mathbf{r})\right] P_{2}(\mathbf{r})+\left[\mathbf{d} P_{1}(\mathbf{r})\right] \wedge \boldsymbol{\Phi}_{1}(\mathbf{r}) \\
& +\left[\mathbf{d} P_{2}(\mathbf{r})\right] \wedge \Phi_{2}(\mathbf{r})-\tau_{1}(\mathbf{r}) P_{1}(\mathbf{r})-\tau_{2}(\mathbf{r}) P_{2}(\mathbf{r})-\tau_{\mathrm{s}}(\mathbf{r}) \\
= & {\left[\mathbf{d} P_{1}(\mathbf{r})\right] \wedge \boldsymbol{\Phi}_{1}(\mathbf{r})+\left[\mathbf{d} P_{2}(\mathbf{r})\right] \wedge \boldsymbol{\Phi}_{2}(\mathbf{r})-\boldsymbol{\tau}_{\mathrm{s}}(\mathbf{r})=0 } \\
\mathbf{d} \wedge \boldsymbol{\Psi}(\mathbf{r})-\gamma & =\left[\mathbf{d} \wedge \boldsymbol{\Psi}_{1}(\mathbf{r})\right] P_{1}(\mathbf{r})+\left[\mathbf{d} \wedge \boldsymbol{\Psi}_{2}(\mathbf{r})\right] P_{2}(\mathbf{r})+\left[\mathbf{d} P_{1}(\mathbf{r})\right] \wedge \boldsymbol{\Psi}_{1}(\mathbf{r}) \\
& +\left[\mathbf{d} P_{2}(\mathbf{r})\right] \wedge \Psi_{2}(\mathbf{r})-\gamma_{1}(\mathbf{r}) P_{1}(\mathbf{r})-\gamma_{2}(\mathbf{r}) P_{2}(\mathbf{r})-\gamma_{\mathrm{s}}(\mathbf{r}) \\
= & {\left[\mathbf{d} P_{1}(\mathbf{r})\right] \wedge \boldsymbol{\Psi}_{1}(\mathbf{r})+\left[\mathbf{d} P_{2}(\mathbf{r})\right] \wedge \boldsymbol{\Psi}_{2}(\mathbf{r})-\gamma_{\mathrm{s}}(\mathbf{r})=0 . }
\end{aligned}
$$

Here we have applied conditions (1) for the systems 1 and 2 to cancel four terms in each equation.

Because the characteristic functions are constant outside the surface $S$, the two one-forms above, denoted compactly as

$$
\mathbf{n}_{1}(\mathbf{r})=\mathbf{d} P_{1}(\mathbf{r}) \quad \mathbf{n}_{2}(\mathbf{r})=\mathbf{d} P_{2}(\mathbf{r})=-\mathbf{n}_{1}(\mathbf{r})
$$

vanish outside the surface $S$. Writing equations (10), (11) as

$$
\begin{aligned}
& \mathbf{n}_{1}(\mathbf{r}) \wedge \Phi_{1}(\mathbf{r})+\mathbf{n}_{2}(\mathbf{r}) \wedge \Phi_{2}(\mathbf{r})=\tau_{\mathrm{s}}(\mathbf{r}) \\
& \mathbf{n}_{1}(\mathbf{r}) \wedge \Psi_{1}(\mathbf{r})+\mathbf{n}_{2}(\mathbf{r}) \wedge \Psi_{2}(\mathbf{r})=\gamma_{\mathrm{s}}(\mathbf{r})
\end{aligned}
$$

the additional electric and magnetic sources $\gamma_{\mathrm{s}}(\mathbf{r}), \boldsymbol{\tau}_{\mathrm{s}}(\mathbf{r})$ are seen to be required for the Maxwell equations to be valid for the combined system, unless the left-hand sides vanish. The additional sources are seen to be surface sources, because the left-hand sides vanish outside $S$. Equations (13) and (14) give the boundary conditions between the fields 1 and 2 at the surface $S$ in symmetric form. This is an aid to memorizing the formula since no preference to the sides 1 and 2 of the surface is made.

One can have a visual picture of the one-forms $\mathbf{n}_{i}(\mathbf{r})=\mathbf{d} P_{i}(\mathbf{r})$ by assuming instead of a sharp surface a layer of finite thickness separating $V_{1}$ and $V_{2}$ by two surfaces $S_{1}$ and $S_{2}$, figure 2. If $P_{1}(\mathbf{r})$ is a function changing continuously from 0 on $S_{2}$ to 1 on $S_{1}, \mathbf{n}_{1}(\mathbf{r})$ is a one-form defined by a set of surfaces of $P_{1}=$ constant. A similar picture can be applied to the one-form $\mathbf{n}_{2}(\mathbf{r})$. When $S_{1}$ and $S_{2}$ approach $S$, the resulting one-forms $\mathbf{n}_{1}(\mathbf{r})$ and $\mathbf{n}_{2}(\mathbf{r})$ consist of infinitely dense sets of constant- $P_{i}$ surfaces in $S$, which can be represented mathematically in terms of a surface-delta function. In the Gibbsian vector formalism one introduces unit vectors normal to the surface which has the drawback that, at any corner points of the surface with a discontinuous tangent, the normal direction is not unique. The one-form counterparts $\mathbf{n}_{i}(\mathbf{r})$ do not have this defect, since they are defined on the surface $S$ without any normal direction. 


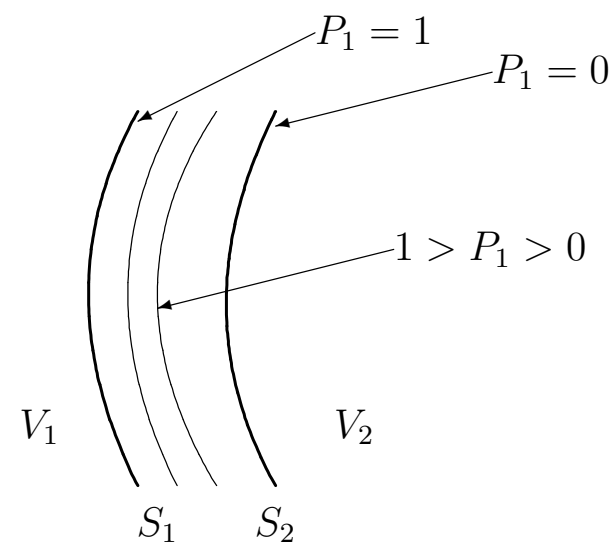

Figure 2. Surface $S$ between regions $V_{1}$ and $V_{2}$ as expanded to a layer. The one-form $\mathbf{d} P_{1}(\mathbf{r})$ is a set of surfaces of constant $P_{1}$ between $S_{1}$ and $S_{2}$.

\section{Boundary conditions}

It was seen that the surface-source three-forms $\tau_{\mathrm{s}}(\mathbf{r}), \gamma_{\mathrm{s}}(\mathbf{r})$ defined by the left-hand sides of equations (13) and (14) are required as a kind of glue to match the two electromagnetic systems together into a combined system. If the original two fields happen to satisfy certain continuity conditions at $S$ (such that the left-hand sides vanish), the surface sources are unnecessary. However, equations (13), (14) can also be understood in the converse way. Assuming that there are given surface sources $\gamma_{\mathrm{s}}(\mathbf{r}), \tau_{\mathrm{s}}(\mathbf{r})$ on a surface $S$, the fields on each side of the surface $S$ must satisfy the conditions (13), (14). If there are no surface sources, i.e. $\gamma_{\mathrm{s}}(\mathbf{r})=0, \tau_{\mathrm{s}}(\mathbf{r})=0$, the fields must satisfy the corresponding continuity equation at $S$.

The conditions (13) and (14) can be expanded in terms of three-dimensional fields and sources when substituting expressions of the form (2) and (3) as

$\mathbf{n}_{1}(\mathbf{r}) \wedge\left[\mathbf{B}_{1}(\mathbf{r})+\mathbf{E}_{1}(\mathbf{r}) \wedge \mathbf{d} t\right]+\mathbf{n}_{2}(\mathbf{r}) \wedge\left[\mathbf{B}_{2}(\mathbf{r})+\mathbf{E}_{2}(\mathbf{r}) \wedge \mathbf{d} t\right]=\varrho_{\mathrm{ms}}(\mathbf{r})-\mathbf{M}_{\mathrm{s}}(\mathbf{r}) \wedge \mathbf{d} t$

$\mathbf{n}_{1}(\mathbf{r}) \wedge\left[\mathbf{D}_{1}(\mathbf{r})-\mathbf{H}_{1}(\mathbf{r}) \wedge \mathbf{d} t\right]+\mathbf{n}_{2}(\mathbf{r}) \wedge\left[\mathbf{D}_{2}(\mathbf{r})-\mathbf{H}_{2}(\mathbf{r}) \wedge \mathbf{d} t\right]=\varrho_{\mathrm{s}}(\mathbf{r})-\mathbf{J}_{\mathrm{s}}(\mathbf{r}) \wedge \mathbf{d} t$.

Separating the terms which contain the one-form $\mathbf{d} t$ (contracting with a time-like vector) we have

$$
\begin{aligned}
& \mathbf{n}_{1}(\mathbf{r}) \wedge \mathbf{B}_{1}(\mathbf{r})+\mathbf{n}_{2}(\mathbf{r}) \wedge \mathbf{B}_{2}(\mathbf{r})=\varrho_{\mathrm{ms}}(\mathbf{r}) \\
& \mathbf{n}_{1}(\mathbf{r}) \wedge \mathbf{E}_{1}(\mathbf{r})+\mathbf{n}_{2}(\mathbf{r}) \wedge \mathbf{E}_{2}(\mathbf{r})=-\mathbf{M}_{\mathrm{s}}(\mathbf{r}) \\
& \mathbf{n}_{1}(\mathbf{r}) \wedge \mathbf{D}_{1}(\mathbf{r})+\mathbf{n}_{2}(\mathbf{r}) \wedge \mathbf{D}_{2}(\mathbf{r})=\varrho_{\mathrm{s}}(\mathbf{r}) \\
& \mathbf{n}_{1}(\mathbf{r}) \wedge \mathbf{H}_{1}(\mathbf{r})+\mathbf{n}_{2}(\mathbf{r}) \wedge \mathbf{H}_{2}(\mathbf{r})=\mathbf{J}_{\mathrm{s}}(\mathbf{r}) .
\end{aligned}
$$

In Gibbsian vector notation, these four conditions correspond to the classical interface conditions for vector fields and scalar or vector surface sources. $\mathbf{n}_{1}, \mathbf{n}_{2}$ now denote vectors normal to the surface $S$ pointing towards media 1 and 2, respectively:

$$
\begin{aligned}
& \mathbf{n}_{1}(\mathbf{r}) \cdot \mathbf{B}_{1}(\mathbf{r})+\mathbf{n}_{2}(\mathbf{r}) \cdot \mathbf{B}_{2}(\mathbf{r})=\varrho_{\mathrm{ms}}(\mathbf{r}) \\
& \mathbf{n}_{1}(\mathbf{r}) \times \mathbf{E}_{1}(\mathbf{r})+\mathbf{n}_{2}(\mathbf{r}) \times \mathbf{E}_{2}(\mathbf{r})=-\mathbf{M}_{\mathrm{s}}(\mathbf{r}) \\
& \mathbf{n}_{1}(\mathbf{r}) \cdot \mathbf{D}_{1}(\mathbf{r})+\mathbf{n}_{2}(\mathbf{r}) \cdot \mathbf{D}_{2}(\mathbf{r})=\varrho_{\mathrm{s}}(\mathbf{r}) \\
& \mathbf{n}_{1}(\mathbf{r}) \times \mathbf{H}_{1}(\mathbf{r})+\mathbf{n}_{2}(\mathbf{r}) \times \mathbf{H}_{2}(\mathbf{r})=\mathbf{J}_{\mathrm{s}}(\mathbf{r}) .
\end{aligned}
$$

The symmetric form of expressing the boundary conditions is effective for memorizing the formulae. 
From equations (13), (14) and (17)-(20) it is clear that the sources defined by the left-hand sides are surface sources since the one-forms $\mathbf{n}_{1}(\mathbf{r})$ and $\mathbf{n}_{2}(\mathbf{r})=-\mathbf{n}_{1}(\mathbf{r})$ vanish outside $S$. Let us study their nature by an example. Taking equation (19), the charge three-form is defined by

$$
\varrho_{\mathrm{s}}(\mathbf{r})=\mathbf{n}_{1}(\mathbf{r}) \wedge \mathbf{D}_{\Delta}(\mathbf{r}) \quad \mathbf{D}_{\Delta}(\mathbf{r})=\mathbf{D}_{1}(\mathbf{r})-\mathbf{D}_{2}(\mathbf{r}) .
$$

The two-form $\mathbf{D}_{\Delta}(\mathbf{r})$ fills all space and $\mathbf{n}_{1}=\mathbf{d} P_{1}(\mathbf{r})$ picks its value at the surface $S$. Considering a point $\mathbf{r}$ on $S$, the one-form $\mathbf{n}_{1}(\mathbf{r})$ defines a local tangent plane, say the $x y$ plane. Hence, it is a multiple of the one-form $\mathbf{d} z$ and satisfies $\mathbf{d} z \wedge \mathbf{n}_{1}=0$ at the point $\mathbf{r}$. Expanding the two-form $\mathbf{D}_{\Delta}$ at $\mathbf{r}$ as

$$
\mathbf{D}_{\Delta}=D_{x} \mathbf{d} y \wedge \mathbf{d} z+D_{y} \mathbf{d} z \wedge \mathbf{d} x+D_{z} \mathbf{d} x \wedge \mathbf{d} y
$$

the charge three-form at the point $\mathbf{r}$ becomes

$$
\varrho_{\mathrm{s}}=\mathbf{n}_{1} \wedge \mathbf{D}_{\Delta}=D_{z} \mathbf{n}_{1} \wedge \mathbf{d} x \wedge \mathbf{d} y .
$$

Thus, the magnitude of $\varrho_{\mathrm{s}}(\mathbf{r})$ is the $D_{z}$ component of the difference two-form $\mathbf{D}_{\Delta}=\mathbf{D}_{1}-\mathbf{D}_{2}$ which multiplies the surface-delta function of $\mathbf{n}_{1}$. Obviously, the other source components can be interpreted in a similar way.

As a special case we may consider the problem when the fields and sources are identically zero in $V_{2}$. In this case, the conditions (13)-(14) become

$$
\begin{aligned}
& \mathbf{n}_{1}(\mathbf{r}) \wedge \Phi_{1}(\mathbf{r})=\tau_{\mathrm{s}}(\mathbf{r}) \\
& \mathbf{n}_{1}(\mathbf{r}) \wedge \Psi_{1}(\mathbf{r})=\gamma_{\mathrm{s}}(\mathbf{r})
\end{aligned}
$$

or, in terms of three-dimensional quantities,

$$
\begin{aligned}
& \mathbf{n}_{1}(\mathbf{r}) \wedge \mathbf{B}_{1}(\mathbf{r})=\varrho_{\mathrm{ms}}(\mathbf{r}) \\
& \mathbf{n}_{1}(\mathbf{r}) \wedge \mathbf{E}_{1}(\mathbf{r})=-\mathbf{M}_{\mathrm{s}}(\mathbf{r}) \\
& \mathbf{n}_{1}(\mathbf{r}) \wedge \mathbf{D}_{1}(\mathbf{r})=\varrho_{\mathrm{s}}(\mathbf{r}) \\
& \mathbf{n}_{1}(\mathbf{r}) \wedge \mathbf{H}_{1}(\mathbf{r})=\mathbf{J}_{\mathrm{s}}(\mathbf{r}) .
\end{aligned}
$$

This means that if we want to terminate an electromagnetic field at a surface $S$, the sources above must be placed on $S$ to cancel the fields penetrating into $V_{2}$.

When medium 2 is perfectly conducting, there are no magnetic charges and magnetic currents. In this case, the conditions (30), (31) become simpler:

$$
\begin{aligned}
& \mathbf{n}_{1}(\mathbf{r}) \wedge \mathbf{B}_{1}(\mathbf{r})=0 \\
& \mathbf{n}_{1}(\mathbf{r}) \wedge \mathbf{E}_{1}(\mathbf{r})=0 .
\end{aligned}
$$

\section{Huygens' principle}

The boundary condition formulae can also be used for deriving Huygens' principle. Assume that the source-field systems 1 and 2 are the same and the sources are all in the region $V_{1}$. In this case, the sources defined by equations (28), (29) create a field in $V_{2}$ which cancels that from the original sources 1 , figure 3. Thus, the surface sources (28), (29) with minus signs added give exactly the same fields in the region $V_{2}$ as the sources 1 . Because the original sources can be replaced by the surface sources

$$
\begin{gathered}
\tau_{\mathrm{H}}(\mathbf{r})=-\mathbf{n}_{1}(\mathbf{r}) \wedge \Phi_{1}(\mathbf{r}) \\
\gamma_{\mathrm{H}}(\mathbf{r})=-\mathbf{n}_{1}(\mathbf{r}) \wedge \Psi_{1}(\mathbf{r})
\end{gathered}
$$

these can be identified with the Huygens sources $[15,16]$, i.e. surface sources equivalent to sources behind the surface. 
<smiles>CCCC([AlH2])C([AlH2])S</smiles>

Figure 3. Huygens' principle replaces sources $\gamma_{1}, \tau_{1}$ in region $V_{1}$ by equivalent sources $\gamma_{\mathrm{H}}, \tau_{\mathrm{H}}$ on surface $S$. Both will produce the same field in region $V_{2}$.

To check this result, let us consider the field radiated by the difference of the original sources and the Huygens sources to see if this vanishes in $V_{2}$. Such a field satisfies the equations

$$
\begin{aligned}
& \mathbf{d} \wedge \Phi_{\mathrm{o}}(\mathbf{r})=\tau_{1}(\mathbf{r})-\tau_{\mathrm{H}}(\mathbf{r})=\tau_{1}(\mathbf{r})+\mathbf{n}_{1}(\mathbf{r}) \wedge \Phi_{1}(\mathbf{r}) \\
& \mathbf{d} \wedge \Psi_{\mathrm{o}}(\mathbf{r})=\gamma_{1}(\mathbf{r})-\gamma_{\mathrm{H}}(\mathbf{r})=\gamma_{1}(\mathbf{r})+\mathbf{n}_{1}(\mathbf{r}) \wedge \boldsymbol{\Psi}(\mathbf{r})
\end{aligned}
$$

Replacing the original sources by

$$
\begin{aligned}
& \tau_{1}(\mathbf{r})=\mathbf{d} \wedge \Phi_{1}(\mathbf{r})=P_{1}(\mathbf{r}) \mathbf{d} \wedge \Phi_{1}(\mathbf{r}) \\
& \gamma_{1}(\mathbf{r})=\mathbf{d} \wedge \Psi_{1}(\mathbf{r})=P_{1}(\mathbf{r}) \mathbf{d} \wedge \Psi_{1}(\mathbf{r})
\end{aligned}
$$

where the $P_{1}(\mathbf{r})$ functions can be inserted because the sources vanish in $V_{2}$, equations (38) and (39) can be expressed as

$$
\begin{aligned}
& \mathbf{d} \wedge \Phi_{\mathrm{o}}(\mathbf{r})=P_{1}(\mathbf{r}) \mathbf{d} \wedge \boldsymbol{\Phi}_{1}(\mathbf{r})+\mathbf{n}_{1}(\mathbf{r}) \wedge \Phi_{1}(\mathbf{r})=\mathbf{d} \wedge\left[P_{1}(\mathbf{r}) \Phi_{1}(\mathbf{r})\right] \\
& \mathbf{d} \wedge \Psi_{\mathrm{o}}(\mathbf{r})=P_{1}(\mathbf{r}) \mathbf{d} \wedge \boldsymbol{\Psi}_{1}(\mathbf{r})+\mathbf{n}_{1}(\mathbf{r}) \wedge \boldsymbol{\Psi}_{1}(\mathbf{r})=\mathbf{d} \wedge\left[P_{1}(\mathbf{r}) \Psi_{1}(\mathbf{r})\right] .
\end{aligned}
$$

If we write these as

$$
\begin{aligned}
& \mathbf{d} \wedge\left[\Phi_{\mathrm{o}}(\mathbf{r})-P_{1}(\mathbf{r}) \Phi_{1}(\mathbf{r})\right]=0 \\
& \mathbf{d} \wedge\left[\Psi_{\mathrm{o}}(\mathbf{r})-P_{1}(\mathbf{r}) \Psi_{1}(\mathbf{r})\right]=0
\end{aligned}
$$

and assume uniqueness of solution for the fields, we can argue that the terms in square brackets must vanish because they have no sources on the right-hand side. This means that the original sources minus the Huygens sources create fields which vanish in $V_{2}$ because they involve the function $P_{1}(\mathbf{r})$. The uniqueness of fields radiated by electromagnetic sources requires certain radiation conditions at infinity. They depend on the specific nature of the electromagnetic medium and will not be discussed further.

\section{Conclusion}

Boundary conditions for electromagnetic fields have been derived using the differential-form formalism in a simple way suitable for presentation to physics or engineering students taking a course on electromagnetism in a less conventional formalism. Transforming the method to the language of Gibbsian vector algebra, the same can be also given to the audience of a more conventional electromagnetics course. Huygens's principle is obtained through the same line of thought without reference to Green functions. 


\section{Acknowledgments}

The present article was prepared while BJ made a three-month visit to the Electromagnetics Laboratory at the Helsinki University of Technology, during the spring term of 1999 . He wishes to express his gratitude to the EM Laboratory for giving him such an opportunity. IVL is indebted to the late Professor Deschamps for the stimulus to study differential forms, during a Fulbright post-doctoral scholarship at the University of Illinois, Urbana-Champaign, in 1973.

\section{References}

[1] Maxwell J C 1891 A Treatise on Electricity and Magnetism 3rd edn (Oxford: Clarendon)

[2] Heaviside O Electrical Papers reprinted 1970 (New York: Chelsea) vol 1, p 447; vol 2, p 172 (original edition, London 1892)

The duplex equations were first published in The Electrician in 1885.

[3] Gibbs J W Element of Vector Analysis privately printed in two parts 1881, 1884 (reprinted 1961 The Scientific Papers of J Willard Gibbs (New York: Dover))

Gibbs J W and Wilson E B 1909 Vector Analysis (New York: Scribner) (reprinted 1960 (New York: Dover))

[4] Flanders H 1963 Differential Forms with Applications to the Physical Sciences (New York: Academic)

[5] Cartan H 1967 Formes Différentielles (Paris: Hermann)

[6] Misner C W, Thorne K S and Wheeler J A 1973 Gravitation (San Francisco: Freeman)

[7] Deschamps G A 1981 Electromagnetics and differential forms Proc. IEEE 69 676-96

[8] Baldomir D 1986 Differential forms and electromagnetism in 3-dimensional Euclidean space $R^{3}$ IEE Proc. A 133 139-43

[9] Hammond P and Baldomir D 1988 Dual energy methods in electromagnetism using tubes and slices IEE Proc. A $135167-72$

[10] Baldomir D 1993 Global geometry of electromagnetic systems IEE Proc. A $140142-50$

[11] Warnick K F, Selfridge R H and Arnold D V 1995 Electromagnetic boundary conditions and differential forms IEE Proc. Microwave Ant. Propagat. 142 326-32

[12] Jancewicz B 1996 A variable metric electrodynamics. The Coulomb and Biot-Savart laws in anisotropic media Ann. Phys. 245 227-4

[13] Warnick K F and Arnold D V 1996 Electromagnetic Green functions using differential forms J. Electromagn. Waves Appl. 10 427-38

[14] Baldomir D and Hammond P 1996 Geometry of Electromagnetic Systems (Oxford: Clarendon)

[15] Lindell I V 1992 Methods for Electromagnetic Field Analysis (Oxford: Oxford University Press) pp 182-7

[16] Baker B B and Copson E T 1939 The Mathematical Theory of Huygens' Principle (Cambridge: Cambridge University Press) 
